\title{
A Randomized Controlled Clinical Study Comparing the Diagnostic Accuracy of the Histologic Prediction for Colorectal Polyps Depending on the Use of Either Magnified or Nonmagnified Narrow Band Imaging
}

\author{
Jin Joo Kim¹, Kyoung Sup Hong ${ }^{2}$, Joo Sung Kim² and Hyun Chae Jung \\ ${ }^{1}$ Division of Gastroenterology, Center for Health Promotion, Seoul National University Hospital, Seoul National University College of \\ Medicine, Seoul, ${ }^{2}$ Department of Internal Medicine and Liver Research Institute, Seoul National University College of Medicine, Seoul, Korea
}

Background/Aims: The aim of this study was to compare the diagnostic capabilities of narrow band imaging (NBI) colonoscopy with and without optical magnification in differentiating neoplastic from nonneoplastic colorectal polyps.

Methods: Between April 2012 and March 2013, 122 patients with colorectal polyps detected by using diagnostic conventional colonoscopy were prospectively enrolled. A total of 236 polyps were evaluated with NBI, in vivo in real time during therapeutic colonoscopy, by one experienced endoscopist. Whether magnification was used or not was determined by randomization. After an in vivo real-time endoscopic prediction of histology, all lesions were endoscopically excised. Surgical pathologic reports were used as the criterion standards. The sensitivity ( $\mathrm{Sn}$ ), specificity (Sp), positive predictive value (PPV), and negative predictive value (NPV) of identifying neoplastic polyps were calculated.

Results: A total of 236 lesions with an average size of $5.6 \mathrm{~mm}$ in 122 patients were assessed (159 neoplastic, 77 nonneoplastic). The Sn, Sp, PPV, and NPV in differentiating neoplastic from nonneoplastic lesions with the magnified NBI were $97.5 \%, 83.3 \%, 94.0 \%$, and $92.6 \%$, respectively, whereas those of the nonmagnified NBI group were $97.5 \%, 85.1 \%, 91.7 \%$, and $95.2 \%$, respectively.

Conclusions: Nonmagnified NBI colonoscopy distinguishes neoplastic from nonneoplastic colorectal polyps as accurately as does magnified NBI colonoscopy. Clin Endosc 2015;48:528-533

Key Words: Narrow band imaging; Magnifying colonoscopy; Histology; Colorectal polyp

\section{INTRODUCTION}

The detection and removal of neoplastic polyps are important because of the well-known relation of these polyps to colorectal cancer. ${ }^{1}$ According to the sequence of adenocarcinoma, it has been generally accepted that colorectal cancer develops

Received: January 2, 2015 Revised: March 12, 2015

Accepted: March 13, 2015

Correspondence: Kyoung Sup Hong

Department of Internal Medicine, Seoul National University Hospital, Seoul National University College of Medicine, 101 Daehak-ro, Jongno-gu, Seoul 03080, Korea

Tel: +82-2-2072-0360, Fax: +82-2-762-9662, E-mail: kshong1@snu.ac.kr

(cc) This is an Open Access article distributed under the terms of the Creative Commons Attribution Non-Commercial License (http://creativecommons.org/ licenses/by-nc/3.0) which permits unrestricted non-commercial use, distribution, and reproduction in any medium, provided the original work is properly cited. from benign adenomas; thus, removing adenomatous polyps is considered to reduce the risk of colorectal cancer development. ${ }^{2}$ Histologically, colorectal polyps are classified as either neoplastic or nonneoplastic. Nonneoplastic polyps include hyperplastic polyps, harmatomas, lymphoid aggregates, and inflammatory polyps, all of which have little malignant potential.

Colonoscopic polypectomy includes hot biopsy, snare polypectomy, and endoscopic mucosal resection. All of these methods are associated with risks of perforation and bleeding. ${ }^{3}$ The rate of complications for this condition has been estimated at $0.4 \%$ to $0.7 \%$ of cases, which is not negligible. In addition to these risks, colonoscopic polypectomy demands a high cost. Ideally, the indication for colonoscopic polypectomy should be limited to neoplastic polyps that have the 
potential to develop into invasive colorectal carcinoma. However, this risky and expensive technique is routinely done for both neoplastic and nonneoplastic polyps during screening colonoscopies because there is no solid tool to confirm the histologic type of those polyps in vivo in real time. Therefore, differentiating between neoplastic and nonneoplastic polyps in vivo in real time can greatly help in avoiding unnecessary polypectomies.

For an endoscopic diagnosis and the differentiation of colorectal polyps into either the neoplastic or nonneoplastic type, there are currently two diagnostic techniques: dye chromoendoscopy and electronic chromoendoscopy. Dye chromoendoscopy is useful in the differentiation of colorectal polyps in real time by using Kudo's classification, and has gained acceptance especially among Japanese endoscopists. ${ }^{4}$ However, it has not been largely accepted in routine clinical practice in other countries because of several reasons, such as the unavailability of high-magnification endoscopes and the additional time and labor requirement for dye application.

On the other hand, narrow band imaging (NBI) is a simple, "push of a button" electronic chromoendoscopy technique that uses optical narrow band light filters for more detailed visualization of the superficial mucosal architecture and vascular pattern. ${ }^{5}$ It is less time consuming than dye chromoendoscopy, as well as easier and more convenient to use. Several studies have shown that NBI can predict polyp histology with moderate to high accuracy. ${ }^{6-8}$ However, the sensitivity and specificity varies according to the level of magnification. Moreover, although magnified NBI is not routinely used in clinical practice because of the nonavailability of magnified endoscopes outside of reference centers, most of the previous studies have usually focused on the diagnostic accuracy of magnified NBI colonoscopy. Only limited studies have shown that nonmagnified NBI is also useful in predicting colon polyp histology. ${ }^{9-11}$ However, most of them compared nonmagnified NBI with white light endoscopy, and used only photographs of colon polyps retrospectively, not in vivo in real time. Therefore, for the clinical application of nonmagnified NBI, more in vivo real-time studies are required.

Thus, we performed our study to compare the efficacy of magnified and nonmagnified NBI in vivo in real time, and also to analyze the factors relevant to misdiagnosis.

\section{MATERIALS AND METHODS}

\section{Study design/setting}

We conducted a prospective, randomized study in patients who had been referred to the Gastroenterology Department of the Seoul National University Hospital between April 2012 and March 2013. All of them were referred to our department for the therapeutic removal of colorectal polyps. The study protocol conformed to the ethical guidelines of the 1975 Declaration of Helsinki. The patients gave informed consent, and the study was approved by Institutional Review Board of Seoul National University Hospital (IRB no. H-1202-080-398).

\section{Participants}

The study group included patients who had been referred for the removal of colorectal polyps. The exclusion criteria were a history of inflammatory bowel disease, colectomy, colon cancer or polyposis syndrome, use of antiplatelet or anticoagulant drugs that precluded the removal of polyps, presence of poor general condition or any other reason to avoid prolonged procedural time, and inability to provide informed consent. Patients with inadequate bowel preparation, a malignant-appearing mass or in whom the cecum could not be reached, or those who underwent a polypectomy but the polyp could not be retrieved for histopathologic analysis, were also excluded.

\section{Colonoscopy procedure}

After undergoing a standard colonic preparation with a polyethylene glycol-based lavage solution, the patients were sedated with intravenous midazolam and meperidine or fentanyl. All colonoscopies were performed by a single experienced endoscopist (HKS). According to the results of randomization, all patients were selected for either magnified NBI colonoscopies or nonmagnified NBI colonoscopies.

All polyps detected during the procedure were documented for their size, location, and morphology (sessile, pedunculated, and flat). The size was estimated through comparison with open biopsy forceps or the sheath of a polypectomy snare placed against the polyp, whereas the location was estimated by using anatomic landmarks.

\section{Prediction and assessment of histology}

The histology of each polyp was predicted according to the superficial mucosal architecture and vascular pattern recently defined by the NICE (NBI International Colorectal Endoscopic) criteria. ${ }^{12}$ Finally, polyps were removed through either cold biopsy or snare electrocautery, and sent for histopathologic analysis. The polyp histology was determined by experienced pathologists who were blind to the endoscopic diagnosis.

\section{Sample size calculation and statistical analysis}

All statistical analyses were performed with SPSS version 15 (SPSS Inc., Chicago, IL, USA). Assuming that the accuracy of nonmagnified NBI in predicting polyp histology was $80 \%$ and that of magnified NBI was $90 \%$, a sample size of 219 polyps 
was required to detect this difference with $80 \%$ power and an $\alpha$ of 0.05 . According to several previous studies, 72 polyps were detected with conventional colonoscopy in 40 patients. Therefore, we assumed that a sample size of 122 patients would be sufficient to detect 219 polyps. The predicted histology and the actual polyp histology were compared to determine the sensitivity, specificity, and accuracy of magnified and nonmagnified NBI. A chi-square test was used to compare the diagnostic characteristics of both magnified and nonmagnified NBI separately. A $p<0.05$ was considered to be statistically significant.

\section{RESULTS}

\section{Patients}

A total of 122 patients were prospectively enrolled and randomized into either the magnified NBI group or the nonmagnified NBI group. The comparison of clinical characteristics between these two groups is described in Table 1. In terms of sex, age, and number of polyps per patient, there were no significant differences between the two groups.

\section{Characteristics of the polyps}

A total of 236 polyps were detected in 122 patients. One

Table 1. Characteristics of Study Patients

\begin{tabular}{lccc} 
Characteristic & $\begin{array}{c}\text { Magnified NBI group } \\
(\boldsymbol{n}=\mathbf{6 1})\end{array}$ & $\begin{array}{c}\text { Non-magnified NBI group } \\
(\boldsymbol{n}=\mathbf{6 1})\end{array}$ & $\begin{array}{c}\boldsymbol{p} \text {-value } \\
\text { Male sex }\end{array}$ \\
Mean age, yr & $44(72.1)$ & $58(77.0)$ & 0.329 \\
No. of polyps & $58.1(32-72)$ & $2.6(1-9)$ & 0.973 \\
\hline
\end{tabular}

Values are presented as number (\%) or median (range).

NBI, narrow band imaging.

Table 2. Characteristics of the Polyps

\begin{tabular}{|c|c|c|c|}
\hline Characteristic & $\begin{array}{l}\text { Magnified NBI group } \\
\qquad(n=110)\end{array}$ & $\begin{array}{l}\text { Non-magnified NBI group } \\
\qquad(n=126)\end{array}$ & $p$-value \\
\hline Mean size, $\mathrm{mm}$ & $5.5(3.0-12.1)$ & $5.8(2.5-12.0)$ & 0.465 \\
\hline Location & & & 0.323 \\
\hline Cecum & $10(9.1)$ & $9(7.1)$ & \\
\hline Ascending colon & $13(11.8)$ & $12(9.5)$ & \\
\hline Transverse colon & $20(18.2)$ & $24(19.0)$ & \\
\hline Descending colon & $13(11.8)$ & $15(11.9)$ & \\
\hline Sigmoid colon & $14(12.7)$ & $24(19.0)$ & \\
\hline Rectum & $40(36.4)$ & $42(33.3)$ & \\
\hline Morphology (Paris type) & & & 0.094 \\
\hline 0-Ip & 89 (80.9) & $96(76.2)$ & \\
\hline 0 -Is & $4(3.6)$ & $5(4.0)$ & \\
\hline 0-IIa & $17(15.5)$ & $25(19.8)$ & \\
\hline Histology & & & 0.235 \\
\hline Neoplastic & $80(72.3)$ & $79(62.7)$ & \\
\hline Low-grade adenoma & $76(69.1)$ & $75(59.5)$ & \\
\hline Sessile serrated adenoma & $2(1.8)$ & $1(0.8)$ & \\
\hline High grade adenoma & $1(0.9)$ & $2(1.6)$ & \\
\hline Non-invasive carcinoma & $1(0.9)$ & $1(0.8)$ & \\
\hline Invasive carcinoma & 0 & 0 & \\
\hline Non-neoplastic & $30(27.3)$ & $47(37.3)$ & \\
\hline
\end{tabular}

Values are presented as median (range) or number (\%).

NBI, narrow band imaging. 
hundred ten polyps were included in the magnified NBI group and 126 in the nonmagnified NBI group (Table 2). In

Table 3. Performance of Predicting Polyp Histology

\begin{tabular}{|c|c|c|}
\hline \multirow{2}{*}{ Variable } & \multicolumn{2}{|c|}{ Histology } \\
\hline & Neoplastic & Non-neoplastic \\
\hline \multicolumn{3}{|l|}{ Magnified NBI group } \\
\hline Endoscopic prediction & 80 & 30 \\
\hline Neoplastic & 78 & 5 \\
\hline Non-neoplastic & 2 & 25 \\
\hline Sensitivity & \multicolumn{2}{|c|}{$97.5(78 / 80)$} \\
\hline Specificity & \multicolumn{2}{|c|}{$83.3(25 / 30)$} \\
\hline PPV & \multicolumn{2}{|c|}{$94.0(78 / 83)$} \\
\hline NPV & \multicolumn{2}{|c|}{$92.6(25 / 30)$} \\
\hline \multicolumn{3}{|l|}{ Non-magnified NBI group } \\
\hline Endoscopic prediction & 79 & 47 \\
\hline Neoplastic & 77 & 7 \\
\hline Non-neoplastic & 2 & 40 \\
\hline Sensitivity & \multicolumn{2}{|c|}{$97.5(77 / 79)$} \\
\hline Specificity & \multicolumn{2}{|c|}{$85.1(40 / 47)$} \\
\hline PPV & \multicolumn{2}{|c|}{$91.7(77 / 84)$} \\
\hline NPV & \multicolumn{2}{|c|}{$95.2(40 / 42)$} \\
\hline
\end{tabular}

Values are presented as number or percentage (number/total number).

NBI, narrow band imaging; PPV, positive predictive value; NPV, negative predictive value. terms of mean size, location, morphology, and histology of the polyps, there were no statistically significant differences between the two groups.

\section{Performance of predicting polyp histology}

In the magnified NBI group, the endoscopist correctly predicted the neoplastic histology in 78 of 80 polyps and the nonneoplastic histology in 25 of 30 polyps. Similarly, in the nonmagnified NBI group, the endoscopist correctly predicted the neoplastic histology in 77 of 79 polyps and the nonneoplastic histology in 40 of 47 polyps. Therefore, as Table 3 shows, the sensitivity, specificity, positive predictive value, and negative predictive value were $97.5 \%, 83.3 \%, 94.0 \%$, and $92.6 \%$, respectively, in the magnified group. On the other hand, the values were $97.5 \%, 85.1 \%, 91.7 \%$, and $95.2 \%$, respectively, in the nonmagnified group.

\section{Analysis of the misdiagnosed lesions}

When analyzing misdiagnosed lesions (Table 4), the size of the polyps was relatively smaller and the anatomic location was mainly on the left side of the colon. Furthermore, most of the misdiagnosed lesions had a flat-type gross morphology.

\section{DISCUSSION}

NBI applies narrow band light filters for a more detailed

Table 4. Analysis of the Misdiagnosed Lesions

\begin{tabular}{|c|c|c|c|}
\hline Variable & Correct diagnosis & Incorrect diagnosis & $p$-value \\
\hline Lesions & 220 & 16 & \\
\hline Mean size, $\mathrm{mm}$ & $8.9(3.0-12.0)$ & $3.8(3.0-5.0)$ & 0.000 \\
\hline Location & & & $0.000^{\mathrm{a})}$ \\
\hline Cecum & $18(8.2)$ & $1(6.3)$ & \\
\hline Ascending colon & $24(10.9)$ & $1(6.3)$ & \\
\hline Transverse colon & $43(19.5)$ & $1(6.3)$ & \\
\hline Descending colon & $27(12.3)$ & $1(6.3)$ & \\
\hline Sigmoid colon & $30(13.6)$ & $8(50.0)$ & \\
\hline Rectum & $78(35.5)$ & $4(25.0)$ & \\
\hline Distribution & & & 0.000 \\
\hline Right side & $85(38.6)$ & $3(18.8)$ & \\
\hline Left side & $135(61.4)$ & $13(81.2)$ & \\
\hline Morphology (Paris type) & & & 0.000 \\
\hline 0-Ip & $174(79.1)$ & $11(68.7)$ & \\
\hline 0 -Is & $5(2.3)$ & $4(25.0)$ & \\
\hline 0-IIa & $41(18.6)$ & $1(6.3)$ & \\
\hline
\end{tabular}

Values are presented as number, median (range), or number (\%).

${ }^{\text {a) }}$ Chi-square test, linear by linear association. 
characterization of vascular structures. Several studies have evaluated the role of NBI for the prediction of colorectal polyp histology, and compared it with chromoendoscopy or conventional endoscopy. ${ }^{7,13-15}$ In these studies, NBI was shown to have a higher accuracy than conventional colonoscopy, but similar to that of chromoendoscopy. ${ }^{7,13}$ However, most of those studies have used NBI with magnification, which is not done widely in clinical practice. Magnification colonoscopes, which have the ability to magnify up to 100 times, are larger in diameter, stiffer, and more expensive than the standard colonoscopes used for routine colonoscopy, and are not widely available. Therefore, if nonmagnified NBI showed comparable accuracy to magnified NBI, it would be more practical and economic to use nonmagnified NBI and to adopt the so-called resect-and-discard strategy.

In the present study, we demonstrated that nonmagnified NBI has a comparable diagnostic accuracy to magnified NBI. Interestingly, probably because they were processed in an in vivo real-time fashion, the polyp size, gross morphology, and location influenced the performance of the endoscopic histologic prediction. These results suggest that an endoscopic diagnosis with nonmagnified NBI colonoscopy could be an alternative method for histologic confirmation. Moreover, in this in vivo real-time setting, other clinical variables, such as polyp size, gross morphology, and location, influenced the performance of the endoscopist's histology prediction. Therefore, when predicting the histology of colorectal polyps, consideration of those clinical variables would be necessary for a higher accuracy.

This study has several limitations. First, only a relatively small number of polyps were included. Thus, definitive conclusions cannot be drawn from these data, and larger studies are warranted to determine whether statistically significant differences between magnified NBI and nonmagnified NBI exist. Secondly, we did not restrict the polyp size to $5 \mathrm{~mm}$ or smaller in this study. This might have led to a classification bias because larger polyps are more likely to be neoplastic. However, most of the included polyps had a size of $<10 \mathrm{~mm}$ in our study, and therefore only a small proportion of polyps were $>10 \mathrm{~mm}$ and the histologic prediction of only those limited polyps would have been influenced by their sizes. Thirdly, this study was conducted with only one experienced endoscopist. In the case of inexperienced endoscopists, the overall accuracy cannot be guaranteed and a histologic prediction of differentiating neoplastic from nonneoplastic polyps is more difficult. Owing to this limitation, more detailed educational tools and a more precise definition of neoplastic polyp features should be standardized. Lastly, among neoplastic polyps, further histologic predictions could not be made. We did not evaluate the performance of endoscopic diagnosis to differ- entiate neoplastic polyps according to their histologic grades, such as low- or high-grade dysplasia and noninvasive or invasive carcinoma.

The strengths of the present study lie in its prospective design and the fact that every endoscopic histologic prediction was done in vivo in real time. In the real world, an endoscopist usually views a detected lesion in real-time fashion by using multiple angles and light modalities at variable distances. Because this type of in vivo real-time endoscopic classification brings the risk of a considerable investigator bias, every endoscopic procedure and histologic prediction in this study was done by a single endoscopist. In addition, the position of the polyps in the colon, their gross morphology, and their size were also known to the endoscopist, which potentially aided in characterization. For example, small polyps in the rectosigmoid are likely to be hyperplastic. Moreover, this type of prediction truly represents the real-world clinical practice because all these clinical factors are inevitably taken into account when predicting the histology of colorectal polyps. Therefore, the results of our study can strengthen the clinical implication of nonmagnified NBI for the endoscopic prediction of colorectal polyp histology.

In conclusion, nonmagnified NBI has a high accuracy in predicting colorectal polyp histology in vivo in real time when integrated with other variables such as the size, gross morphology, and location of polyps. Therefore, instead of magnified NBI, nonmagnified NBI might be considered a more convenient and practical tool for the in vivo real-time histologic prediction of colorectal polyps. Moreover, incorporating other clinical variables, such as polyp size, gross morphology, and location, into the diagnostic criteria would make the endoscopic histology diagnosis more practical and accurate.

\section{Conflicts of Interest}

The authors have no financial conflicts of interest.

\section{REFERENCES}

1. Vogelstein B, Fearon ER, Hamilton SR, et al. Genetic alterations during colorectal-tumor development. N Engl J Med 1988;319:525-532.

2. Winawer SJ, Zauber AG, Ho MN, et al. Prevention of colorectal cancer by colonoscopic polypectomy. The National Polyp Study Workgroup. N Engl J Med 1993;329:1977-1981.

3. Levin TR, Zhao W, Conell C, et al. Complications of colonoscopy in an integrated health care delivery system. Ann Intern Med 2006;145:880886.

4. Kudo S, Tamura S, Nakajima T, Yamano H, Kusaka H, Watanabe H. Diagnosis of colorectal tumorous lesions by magnifying endoscopy. Gastrointest Endosc 1996;44:8-14

5. Gono K, Obi T, Yamaguchi M, et al. Appearance of enhanced tissue features in narrow-band endoscopic imaging. J Biomed Opt 2004;9:568577.

6. Tischendorf JJ, Wasmuth HE, Koch A, Hecker H, Trautwein C, Winograd R. Value of magnifying chromoendoscopy and narrow band 
imaging (NBI) in classifying colorectal polyps: a prospective controlled study. Endoscopy 2007;39:1092-1096.

7. Chiu HM, Chang CY, Chen CC, et al. A prospective comparative study of narrow-band imaging, chromoendoscopy, and conventional colonoscopy in the diagnosis of colorectal neoplasia. Gut 2007;56:373-379.

8. East JE, Suzuki N, Bassett P, et al. Narrow band imaging with magnification for the characterization of small and diminutive colonic polyps: pit pattern and vascular pattern intensity. Endoscopy 2008;40:811-817.

9. Su MY, Hsu CM, Ho YP, Chen PC, Lin CJ, Chiu CT. Comparative study of conventional colonoscopy, chromoendoscopy, and narrow-band imaging systems in differential diagnosis of neoplastic and nonneoplastic colonic polyps. Am J Gastroenterol 2006;101:2711-2716.

10. Rogart JN, Jain D, Siddiqui UD, et al. Narrow-band imaging without high magnification to differentiate polyps during real-time colonoscopy: improvement with experience. Gastrointest Endosc 2008;68:11361145.

11. Sikka S, Ringold DA, Jonnalagadda S, Banerjee B. Comparison of white light and narrow band high definition images in predicting colon polyp histology, using standard colonoscopes without optical magnification. Endoscopy 2008;40:818-822.

12. Hewett DG, Kaltenbach T, Sano Y, et al. Validation of a simple classification system for endoscopic diagnosis of small colorectal polyps using narrow-band imaging. Gastroenterology 2012;143:599.e1-607.el.

13. Machida H, Sano Y, Hamamoto Y, et al. Narrow-band imaging in the diagnosis of colorectal mucosal lesions: a pilot study. Endoscopy 2004;36:1094-1098.

14. Rastogi A, Bansal A, Wani S, et al. Narrow-band imaging colonoscopy: a pilot feasibility study for the detection of polyps and correlation of surface patterns with polyp histologic diagnosis. Gastrointest Endosc 2008;67:280-286

15. Rastogi A, Pondugula K, Bansal A, et al. Recognition of surface mucosal and vascular patterns of colon polyps by using narrow-band imaging: interobserver and intraobserver agreement and prediction of polyp histology. Gastrointest Endosc 2009;69(3 Pt 2):716-722. 